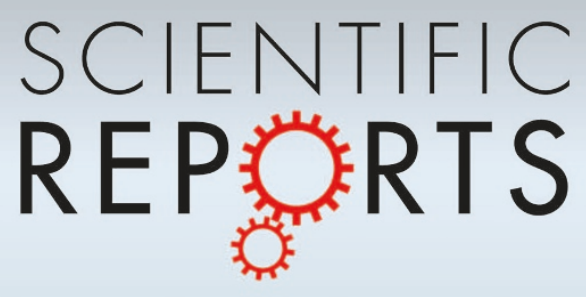

OPEN

SUBJECT AREAS:

NONLINEAR OPTICS

ULTRAFAST PHOTONICS

RAMAN SPECTROSCOPY

MULTIPHOTON MICROSCOPY

Received

26 February 2013

Accepted

23 April 2013

Published

30 May 2013

Correspondence and requests for materials should be addressed to

A.M.Z. (zheltikov@ physics.msu.ru)

\section{The phase-controlled Raman effect}

\author{
A. A. Lanin ${ }^{1,2}$, I. V. Fedotov ${ }^{1,2}$, A. B. Fedotov ${ }^{1,2}$, D. A. Sidorov-Biryukov ${ }^{1,2} \&$ A. M. Zheltikov ${ }^{1,2,3}$
}

'International Laser Center, Physics Department M. V. Lomonosov MSU, Moscow, Russia, ${ }^{2}$ Russian Quantum Center, Novaya 100,
143025 Skolkovo, Moscow Region, Russia, ${ }^{3}$ Department of Physics and Astronomy, Texas A\&M University, College Station TX, 77843-4242 USA.

Unlike spontaneous Raman effect, nonlinear Raman scattering generates fields with a well-defined phase, allowing Raman signals from individual scatterers to add up into a highly directional, high-brightness coherent beam. Here, we show that the phase of coherent Raman scattering can be accurately controlled and finely tuned by using spectrally and temporally tailored optical driver fields. In our experiments, performed with spectrally optimized phase-tunable laser pulses, such a phase control is visualized through the interference of the coherent Raman signal with the field resulting from nonresonant four-wave mixing. This interference gives rise to Fano-type profiles in the overall nonlinear response measured as a function of the delay time between the laser pulses, featuring a well-resolved destructive-interference dip on the dark side of the Raman peak. This phase-control strategy is shown to radically enhance the coherent response from weak Raman modes, thus helping confront long-standing challenges in nonlinear Raman imaging and microspectroscopy.

$\mathrm{N}$ onlinear Raman scattering ${ }^{1-5}$ is one of the key effects in ultrafast optical science, which finds growing applications in ultrashort pulse generation ${ }^{6}$, bioimaging ${ }^{7-9}$, biothreat detection ${ }^{10}$, remote sensing ${ }^{11}$, as well as time-resolved studies on femto- ${ }^{9}$ and attosecond ${ }^{12}$ time scales. In contrast to the spontaneous regime of Raman scattering ${ }^{13}$, where the phase of the scattered field is random, effects related to the phase of the scattered signal in nonlinear versions of Raman scattering are of paramount importance. The phase of the nonlinear Raman signal manifests itself in spectral line shapes of coherent Raman scattering ${ }^{2,3}$ and enters as an important parameter in the nonlinear dynamics of ultrashort optical waveforms ${ }^{6}$ and pulse-shape optimization for enhanced signal-to-background ratio in nonlinear Raman imaging and spectroscopy ${ }^{14}$. The phase effects in coherent Raman scattering enable coherent Raman ellipsometry ${ }^{4}$, optical heterodyne detection ${ }^{3}$, and signal-tobackground enhancement. Optimally shaped field waveforms, chirped light pulses, and time-ordered pulse sequences have been intensely used in ultrafast Raman photonic technologies in the past few years, offering powerful tools for a quantum control of Raman excitation ${ }^{15}$, tailoring of spectral and temporal transformations of ultrashort pulses ${ }^{16}$, coherent Raman spectroscopy with a high spectral resolution ${ }^{17-19}$, and single-beam nonlinear Raman imaging with suppressed nonresonant background $d^{5,20}$.

In this work, we demonstrate that tailored optical driver fields enable a smooth tuning of the phase of the coherent Raman response, giving rise to a rich variety of interference effects, which can dramatically modify the nonlinear dynamics of ultrashort pulses and offer attractive solutions to long-standing issues in Raman imaging and spectroscopy. The nonlinear response measured as a function of the delay time between the chirped pulses forming an optical driver in our experiments is shown to feature a Fano-type profile with a well-resolved destructive-interference dip on the dark side of the Raman peak, visualizing a phase-controlled interference of the coherent Raman signal and the field generated through four-wave mixing (FWM).

\section{Results}

The idea of phase-controlled coherent Raman scattering is illustrated in Figs. 1 and 2. Here, a probe field with a central frequency $\omega_{\mathrm{pr}}$ is inelastically scattered off a Raman vibration of frequency $\Omega_{\mathrm{R}}$, coherently driven by a spectrally and temporally tailored optical driving field, giving rise to a coherent anti-Stokes Raman scattering (CARS) signal at the frequency $\omega_{\mathrm{as}}=\omega_{\mathrm{pr}}+\Omega_{\mathrm{R}}$. For efficient excitation of the Raman mode, the optical driving field should include sufficiently intense spectral components with frequencies $\omega_{\mathrm{pu}}$ and $\omega_{\mathrm{st}}=\omega_{\mathrm{pu}}-\Omega_{\mathrm{R}}$. In the canonical CARS arrangement, such a resonant excitation of a Raman mode is provided by applying spectrally isolated pump and Stokes fields with frequencies $\omega_{\text {pu }}$ and $\omega_{\text {st. }}$. In our scheme (Fig. 1a), the optical driver includes a pair of broadband laser pulses with an identical linear chirp $\varphi(t)=\alpha t^{2}$ and central frequencies $\omega_{\text {pu }}\left(\eta_{1}\right)=\omega_{1}+$ $2 \alpha \eta_{1}$ and $\omega_{\mathrm{st}}\left(\eta_{2}\right)=\omega_{2}+2 \alpha \eta_{2}$, where $\eta_{1}$ and $\eta_{2}=\eta_{1}+\tau$ stand for the time measured in the frames of reference moving with the laser pulses and $\tau$ is the delay time between the laser pulses. The spectrum of the optical driver 

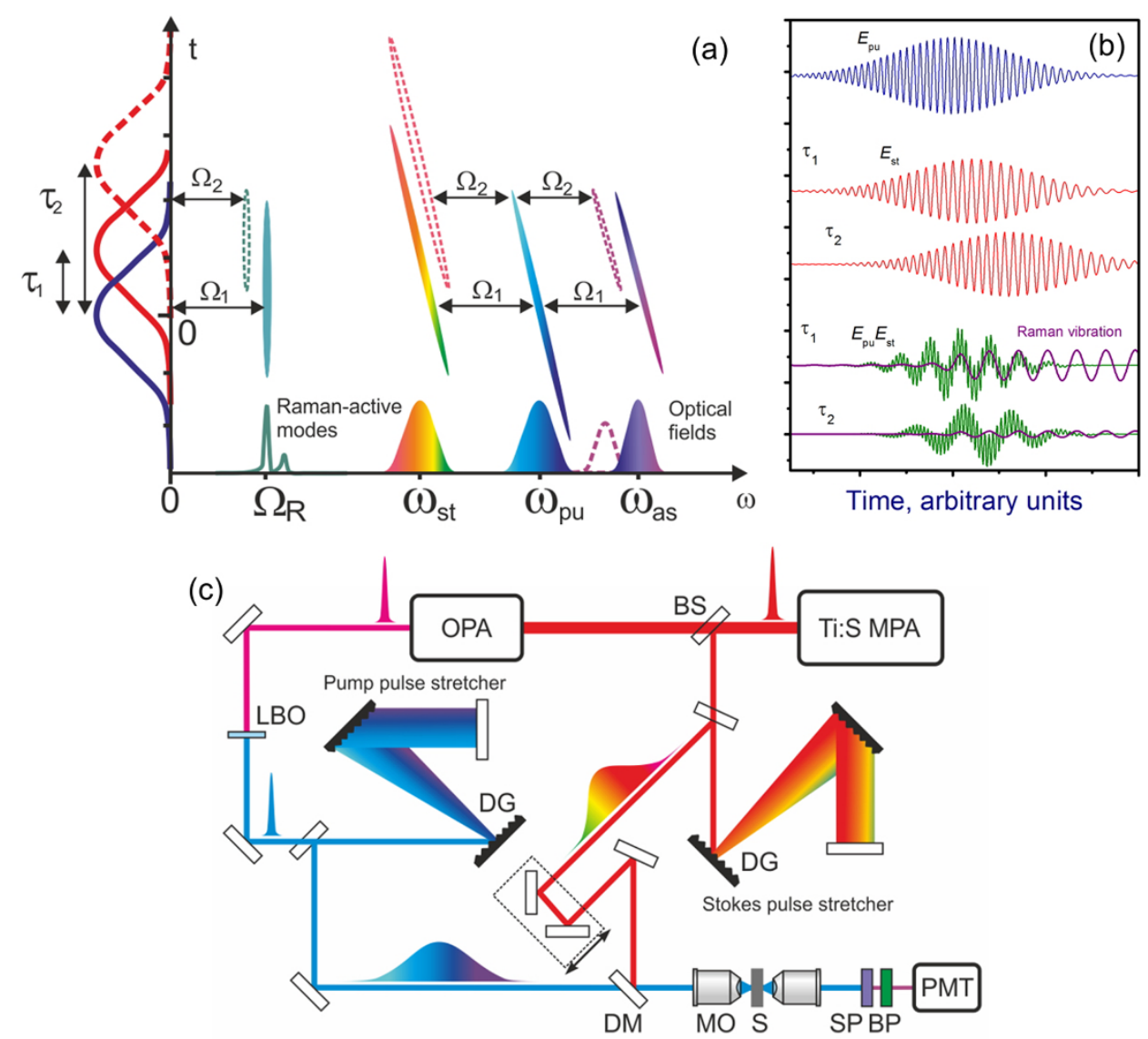

Figure 1 Coherent Raman scattering of tailored optical waveforms: (a) the spectral-temporal map of the optical driver and anti-Stokes fields and (b) the pump (blue) and Stokes (red) fields, the optical driver $A_{p u} A_{S t}^{*}$ (green), and the coherent Raman response (purple). The optical driver, consisting of two linearly chirped pulses with equal chirp parameters and central frequencies $\omega_{\text {pu }}$ and $\omega_{\text {st }}$ (shown in panel (a)), is used for coherent excitation of a Raman-active mode with a frequency $\Omega_{\mathrm{R}}$. Due to the chirp of the laser pulses, the modulation frequency $\Omega_{\mathrm{m}}$ of the optical driver can be scanned by varying the delay time $\tau$ (as shown in panel (b)). The delay times $\tau_{1}$ and $\tau_{2}$ in panels (a) and (b) correspond to modulation frequencies $\Omega_{1}$ and $\Omega_{2}$. With the delay time between the pulses chosen in such a way (time delay $\tau_{1}$ in panels (a) and (b)) that the modulation frequency of the optical driver is tuned to a resonance with the frequency of the Raman mode, $\Omega_{\mathrm{m}}=\Omega_{\mathrm{R}}$, the Raman vibration is driven with a maximum efficiency (panel (b)).

The linearly chirped pulse with the central frequency $\omega_{\mathrm{pr}}=\omega_{\mathrm{pu}}$ and chirp parameter $\alpha$ also serves as a probe field, giving rise to a chirped anti-Stokes signal centered at $\omega_{\mathrm{as}}=\omega_{\mathrm{pr}}+\Omega_{\mathrm{R}}=2 \omega_{\mathrm{pu}}-\omega_{\mathrm{st}}$ (panel (a)). (c) Experimental setup: Ti:S MPA, Ti: Sapphire laser consisting of a mode-locked master oscillator and a multipass amplifier; OPA, optical parametric amplifier; BS, beam splitter, LBO, lithium triborate crystal; DG, diffraction grating; DM, dichroic mirror; MO, microscope objective; S, sample, SP, short-pass filter; BP, band-pass filter; PMT, photomultiplier tube.

consisting of such pulses features a harmonic modulation at the frequency $\Omega_{\mathrm{m}}(\tau)=\omega_{1}-\omega_{2}-2 \alpha \tau$, controlled by the delay time between the pulses $\tau$ (Fig. 1b). As the modulation frequency $\Omega_{\mathrm{m}}$ is scanned through a resonance with the frequency $\Omega_{\mathrm{R}}$ by varying the delay time $\tau$ (Figs. 1a, 1b), the phase of a Raman vibration displays a rapid phase shift by $2 \pi$ (the dash-dotted line in the upper panel in Fig. 2a) as a part of a typical phase response of a harmonic oscillator to a resonant driving force. Experimental examples presented below in this paper and illustrated by Fig. 3 show that more complex phase profiles can be synthesized by coherently combining the interfering responses from several Raman modes with close vibration frequencies.

The overall nonlinear signal measured in experiments, $E=E_{\mathrm{r}}+$ $E_{\mathrm{nr}}$, is a mixture of the coherent Raman signal $E_{\mathrm{r}}$ and a coherent background $E_{\mathrm{nr}}$, originating from nonresonant FWM processes due to the electronic part of optical nonlinearity and off-resonance molecular vibrations. In CARS microspectroscopy and imaging, this nonresonant background has long been viewed as a fundamental limitation on the sensitivity of coherent Raman techniques, strongly motivating the search for effective means for its suppression through carefully optimized polarization arrangements ${ }^{2,4}$, schemes with timedelayed probe pulses ${ }^{14}$, and pulse-shaping approaches ${ }^{5}$. On the other hand, nonresonant coherent Raman scattering is known to provide a constant-phase component of the overall coherent Raman signal, which helps retrieve the phases of Raman-active modes ${ }^{3,4,21}$ and resolve overlapping Raman responses from complex molecules ${ }^{22,23}$, offering elegant solutions for phase-contrast microscopy ${ }^{24}$ and multimodal Raman imaging ${ }^{25-27}$.

Our experimental approach employs an optical driver consisting of a pair of chirped pulses, which has been used earlier for coherent Raman spectroscopy ${ }^{17-19,26-28}$, to demonstrate a smooth phase tunability of coherent Raman scattering. Unlike the earlier work where pulse shaping was used to efficiently suppress the nonresonant background in CARS, in our scheme, the nondispersive coherent background resulting from nonresonant FWM is used to visualize the tunable phase of the coherent Raman response from molecular vibrations driven by a pair of chirped laser pulses. To explain this approach, we use standard approximations to represent the overall nonlinear signal as $E(\tau, z) \propto i \gamma \int_{-\infty}^{\infty} d t \int_{0}^{z} d \xi A_{p r}(t, \xi) \int_{-\infty}^{t} h(t-\theta)$ $A_{p u}(\theta, \xi) A_{S t}^{*}(\theta-\tau, \xi) d \theta$, where $A_{\mathrm{pu}}, A_{\mathrm{St}}$, and $A_{\mathrm{pr}}$ are the complex amplitudes of the pump, probe, and Stokes fields, $\gamma$ is the nonlinear coefficient, and the nonlinear response $h(\theta)=R(\theta)+S(\theta)$ includes the resonant, inertial term, $R(\theta)$, related to Raman modes, along with 

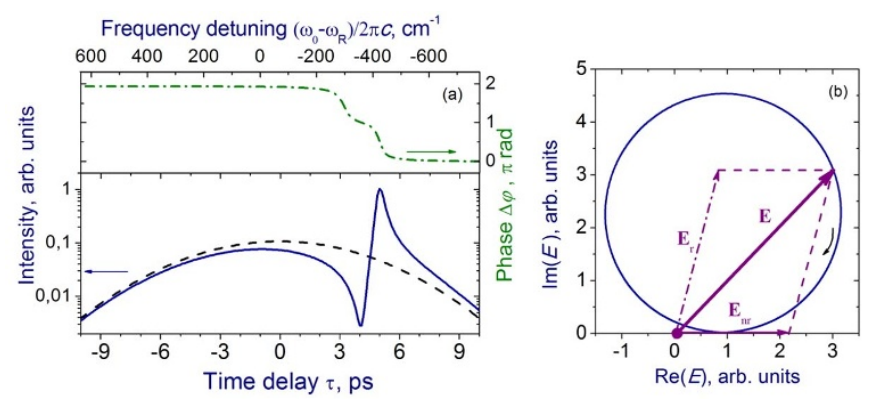

Figure $2 \mid$ Phase-controlled coherent Raman scattering by an isolated harmonic oscillator with $\Omega / 2 \pi \mathrm{c}=2248 \mathrm{~cm}^{-1}, \Gamma / 2 \pi \mathrm{c}=15 \mathrm{~cm}^{-1}$, and $f_{\mathrm{R}}$ $=0.124$. (a) Intensity of the overall coherent signal as a function of the delay time $\tau$ (solid line), intensity of the nonresonant component of the coherent response (dashed line), and the phase shift of the coherent Raman signal relative to the nonresonant background (dash-dotted line) and (b) the trajectory traced by the complex amplitude of the overall coherent signal as a function of the delay time $\tau$ in the plane where the abscissa and ordinate axes are chosen in such a way as to represent the real and imaginary parts of the overall signal. Direction of increasing $\tau$ is shown by an arrow. The optical driver consists of two linearly chirped pulses with $\alpha$ $\approx 3.6 \mathrm{ps}^{-2}$. Parameters of a harmonic oscillator are chosen in such a way to mimic experiments with the $2248-\mathrm{cm}^{-1}$ Raman mode of acetonitrile.

the off-resonance, instantaneous part, $S(\theta)=\bar{f} \delta(\theta), \delta(\theta)$ being the delta function. When the phase mismatch is negligible within the beam-interaction length $L$, the resonant and nonresonant parts of the nonlinear signal are given by $E_{r}(\tau) \propto i \gamma L \int_{-\infty}^{\infty} d t A_{p r}(t) \int_{-\infty}^{t} R$ $(t-\theta) A_{p u}(\theta) A_{S t}^{*}(\theta-\tau) d \theta$ and $E_{n r}(\tau) \propto i \gamma L \int_{-\infty}^{\infty} A_{p r}(t) A_{p u}(t) A_{S t}^{*}(t-$ $\tau) d t$. It is straightforward to see now that the product $A_{p u} A_{S t}^{*}$ serves as an optical driver, providing a coherent excitation of the Raman modes. The Raman response $E_{\mathrm{r}}(\tau)$ is measured against a coherent background $E_{\mathrm{nr}}(\tau)$ (Fig. 2a), which recovers, as can be seen from the expressions above, the cross-correlation trace of the pump, Stokes, and probe pulses.

\section{Discussion}

In our experiments performed within a broad class of liquid-phase and solid-film samples featuring the Raman response of a variable complexity, the overall nonlinear signal measured as a function of the delay time $\tau$ displays a well-resolved Fano-type profile (Fig. 3), as a universal indication (Fig. 2a) of the interference of a signal with a steep dependence on $\tau$, which is due to the coherent Raman field in our case, with a slowly varying signal originating from nonresonant FWM. For large $|\tau|$, the waveform of the overall nonlinear signal (the filled circles in Figs. 3a, 3c) asymptotically tends to the crosscorrelation trace of the laser pulses (the open circles in Figs. 3a, 3c), in agreement with our theoretical analysis (solid and dashed lines in Figs. 2a, 3a, 3c).

The waveforms of the time-resolved nonlinear signals in all our experiments are adequately explained in terms of a dampedoscillator model of Raman vibrations (cf. filled circles and solid lines in Figs. 3a, 3c), translating into Lorenztian spectral profiles for the Raman line shapes, giving $R(\theta)=\sum_{j}^{N} f_{j} \eta_{1 j}^{-1} \eta_{2 j}^{-2}\left(\eta_{1 j}+\eta_{2 j}\right) \sin (\theta /$ $\left.\eta_{1 j}\right) \exp \left(-\theta / \eta_{2 j}\right)$ and $\sum_{j} f_{j}=1-\bar{f}$ for a manifold of $N$ Raman modes with frequencies $\Omega_{j}=2 \pi / \eta_{1 j}$, decay times $\eta_{2 j}=2 / \Gamma_{j}\left(\Gamma_{j}\right.$ is the relevant Raman linewidth), and weighing factors $f_{j}$. In the case of acetonitrile, an accurate fit is achieved (filled circles and solid line in Fig. 3a) with a Raman function including $N=2$ Raman modes with ${ }^{29}$ $\Omega_{1} / 2 \pi \mathrm{c}=2248 \mathrm{~cm}^{-1}, \Gamma_{1} / 2 \pi \mathrm{c}=15 \mathrm{~cm}^{-1}, f_{1}=0.124$ and $\Omega_{2} / 2 \pi \mathrm{c}=$ $2289 \mathrm{~cm}^{-1}, \Gamma_{2} / 2 \pi \mathrm{c}=12 \mathrm{~cm}^{-1}, f_{2}=0.02$. For polystyrene, $N=4$
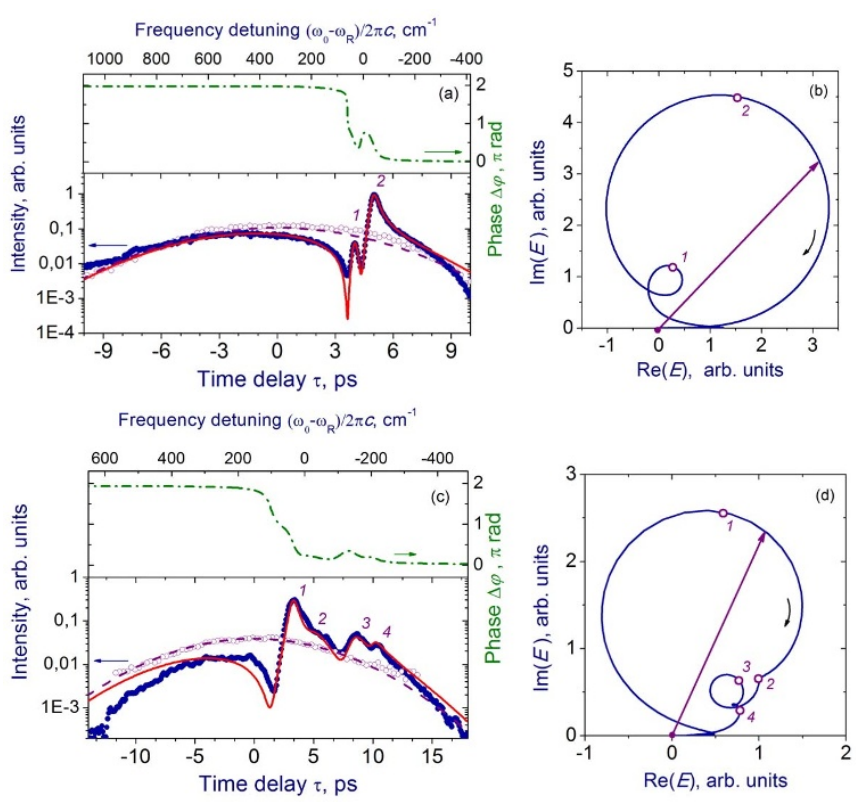

Figure 3 Phase-controlled coherent Raman scattering in acetonitrile (a, b) and polystyrene (c, d). (a, c) Intensity of the overall coherent signal measured (filled circles) and calculated (solid line) as a function of the delay time $\tau$, cross-correlation trace of the laser pulses (open circles) and the intensity of the nonresonant component of the coherent response (dashed line), and the phase shift of the coherent Raman signal relative to the nonresonant background. (b, d) Trajectories traced by the complex amplitude of the overall coherent field in the plane where the abscissa and ordinate axes are chosen in such a way as to represent the real and imaginary parts of the overall signal. Direction of increasing $\tau$ is shown by an arrow. The optical driver consists of two linearly chirped pulses with $\alpha$ $\approx 3.6 \mathrm{ps}^{-2}$. The Raman resonances are labeled with the numbers. Modeling was performed with $\Omega_{1} / 2 \pi \mathrm{c}=2248 \mathrm{~cm}^{-1}, \Gamma_{1} / 2 \pi \mathrm{c}=15 \mathrm{~cm}^{-1}, f_{1}=0.124$, $\Omega_{2} / 2 \pi \mathrm{c}=2289 \mathrm{~cm}^{-1}, \Gamma_{2} / 2 \pi \mathrm{c}=12 \mathrm{~cm}^{-1}, f_{2}=0.02$ and for acetonitrile and $\Omega_{1} / 2 \pi c=2852 \mathrm{~cm}^{-1}, \Gamma_{1} / 2 \pi \mathrm{c}=30 \mathrm{~cm}^{-1}, f_{1}=0.045 ; \Omega_{2} / 2 \pi c=2904 \mathrm{~cm}^{-1}$, $\Gamma_{2} / 2 \pi c=55 \mathrm{~cm}^{-1}, f_{2}=0.17 ; \Omega_{3} / 2 \pi c=3007 \mathrm{~cm}^{-1}, \Gamma_{3} / 2 \pi \mathrm{c}=65 \mathrm{~cm}^{-1}, f_{3}$ $=0.09$; and $\Omega_{4} / 2 \pi \mathrm{c}=3054 \mathrm{~cm}^{-1}, \Gamma_{4} / 2 \pi \mathrm{c}=25 \mathrm{~cm}^{-1}, f_{4}=0.18$ for polystyrene.

Raman modes need to be included for an adequate fit (filled circles and solid line in Fig. 3c) with ${ }^{29} \Omega_{1} / 2 \pi c=2852 \mathrm{~cm}^{-1}, \Gamma_{1} / 2 \pi \mathrm{c}=$ $30 \mathrm{~cm}^{-1}, f_{1}=0.045 ; \Omega_{2} / 2 \pi c=2904 \mathrm{~cm}^{-1}, \Gamma_{2} / 2 \pi c=55 \mathrm{~cm}^{-1}, f_{2}$ $=0.17 ; \Omega_{3} / 2 \pi c=3007 \mathrm{~cm}^{-1}, \Gamma_{3} / 2 \pi \mathrm{c}=65 \mathrm{~cm}^{-1}, f_{3}=0.09$; and $\Omega_{4} /$ $2 \pi \mathrm{c}=3054 \mathrm{~cm}^{-1}, \Gamma_{4} / 2 \pi \mathrm{c}=25 \mathrm{~cm}^{-1}, f_{4}=0.18$. Through this fitting procedure, the entire temporal profile of the phase shift of the coherent Raman signal relative to the nonresonant signal can be retrieved. In the regime where the optical driver is tailored to provide a nearly resonant excitation of the Raman mode, the behavior of this phase closely follows the universal behavior of the phase response function of a generic harmonic oscillator driven with a nearly resonant external force (the dash-dotted lines in the upper panels of Figs. 2a, 3a, 3c).

To show how the nonresonant component of the nonlinear signal can be used to store the phase of the coherent Raman response, it is instructive to represent the complex amplitude of the overall nonlinear signal as a vector $\mathbf{E}$ in a plane where the abscissa and ordinate axes are chosen in such a way ${ }^{30,31}$ as to represent the real and imaginary parts of the field $E$ (Figs. $2 \mathrm{~b}, 3 \mathrm{~b}, 3 \mathrm{~d}$ ). The vector $\mathrm{E}$ is a sum of the vectors $E_{r}$ and $E_{n r}$, representing the amplitudes of the resonant and nonresonant signals (Fig. 2b). The amplitude of the nonresonant part of the signal is real and is represented by a vector $\mathrm{E}_{\mathrm{nr}}$ that is always parallel to the abscissa axis (Fig. 2b). The resonant response $E_{\mathrm{r}}$, on the other hand, displays characteristic rapid phase shifts as the 
Raman shift, $\mathrm{cm}^{-1}$
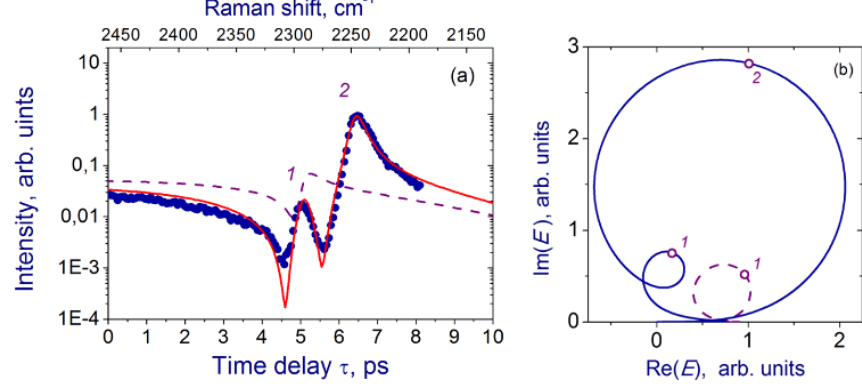

Figure $4 \mid$ Resolving the coherent Raman signal on a finer time scale using an optical drivier consisting of chirped laser pulses with a smaller chirp, $\alpha \approx 2.6 \mathrm{ps}^{-2}$. (a) intensity and (b) the phase trajectory of the overall coherent signal measured (filled circles) and calculated (solid and dashed line) as a function of the delay time $\tau$ with the Raman response of acetonitrile including both the $2248 \mathrm{~cm}^{-1}$ and $2289 \mathrm{~cm}^{-1}$ Raman modes (solid line) and only the $2289 \mathrm{~cm}^{-1}$ mode (dashed line). The Raman resonances are labeled with the numbers.

delay time $\tau$ is scanned through the values corresponding to a resonance of the optical driver with one of the Raman modes. Exactly on resonance, the vector $\mathrm{E}$ is perpendicular to its phase trajectory and its length reaches its maximum. In the case of an isolated Raman mode, the end of the $\mathrm{E}$ vector moves along an almost circular trajectory (Fig. 2b). When more than one Raman vibration contribute to the nonlinear response, the trajectory traced by the $\mathrm{E}$ vector becomes more complicated, with every Raman mode giving rise to a cycloidlike feature in this trajectory (Figs. 3b, 3d). For acetonitrile, such features are seen at delay times $\tau_{1} \approx 4.0 \mathrm{ps}$ and $\tau_{2} \approx 5.0 \mathrm{ps}$, shown by the numbers in Figs. $3 \mathrm{a}$ and $3 \mathrm{~b}$. In the case of polystyrene, four such features are observed at $\tau_{1} \approx 3.2 \mathrm{ps}, \tau_{2} \approx 5.2 \mathrm{ps}, \tau_{3} \approx 8.6 \mathrm{ps}$, and $\tau_{4} \approx 10.3$ ps (Figs. 3c, 3d), corresponding to the four Raman modes excited by the shaped optical driver field.

While the existing Raman methods primarily rely on the detection of the main peak in the signal of Raman scattering, experiments presented here suggest that the detection of the entire Raman response, including the dark signal in Figs. 3a, 3c, can help confront the long-standing issue of coherent background in coherent Raman imaging and spectroscopy. As can be seen from experimental results presented in Fig. 3a, destructive interference between the coherent Raman signal and the nonresonant FWM field can reduce the overall coherent signal in the region of the interference-dip area of the Fano profile, allowing a background-suppressed detection of weak Raman modes. To demonstrate this possibility, measurements on the weak Raman mode at $\Omega_{2} / 2 \pi \mathrm{c}=2289 \mathrm{~cm}^{-1}$ were performed using an optical driver consisting of a pair of chirped laser pulses with the same central wavelengths as before, but with a smaller chirp parameter, $\alpha \approx 2.6 \mathrm{ps}^{-2}$, providing slower variations of the instantaneous frequencies inside the laser pulses, thus allowing ultrafast features of the time-resolved Raman response to be enlarged on an effectively finer time scale. With this experimental arrangement, the peak corresponding to the $\Omega_{2} / 2 \pi \mathrm{c}=2289 \mathrm{~cm}^{-1}$ Raman is detected against the overall coherent signal with a signal-to-background ratio in excess of 20 (Fig. 4a). Detection of this Raman mode with such a high contrast becomes possible due to a destructive interference of the coherent response of the stronger, Raman mode at $\Omega_{1} / 2 \pi \mathrm{c}=$ $2248 \mathrm{~cm}^{-1}$ and the FWM signal (Figs. 4a, 4b). Without the $\Omega_{1}$ Raman vibration, the response of the $\Omega_{2}$ Raman mode would have appeared as a weak dispersive feature with a contrast of less than 6 (the dashed line in Fig. 4a). The signal contrast for the $\Omega_{2}$ mode can be further enhanced by another order of magnitude, as the theoretical limit for this effect (the solid line in Fig. 4a), by using laser pulses with smaller $\alpha$.
To summarize, we have shown that spectrally and temporally tailored optical driver fields enable a phase control of coherent Raman scattering. In experiments with an optical driver consisting of a pair of chirped laser pulses, such a phase control has been visualized through the interference of the coherent Raman signal with the field resulting from nonresonant FWM. This interference gives rise to Fano-type profiles in the overall nonlinear response measured as a function of the delay time between the chirped laser pulses forming an optical drive. Phase-controlled Raman scattering can dramatically modify the nonlinear dynamics of ultrashort pulses and help confront long-standing challenges in nonlinear Raman imaging and microspectroscopy.

\section{Methods}

In our experiments, an optical driver consists of the second-harmonic output of a Ti: sapphire-laser-pumped optical parametric amplifier (OPA) with a wavelength $\lambda_{1}=$ $2 \pi c / \omega_{1}$ tunable from 630 to $720 \mathrm{~nm}$ and a Ti: sapphire laser output with a central wavelength $\lambda_{2}=2 \pi c / \omega_{2} \approx 808 \mathrm{~nm}$. The second-harmonic field with $\lambda_{1}$ also serves as a probe field, giving rise to a linearly chirped anti-Stokes signal centered at $\lambda_{\mathrm{a}}=2 \pi \mathrm{c}$ l $\omega_{\mathrm{a}}$, with $\omega_{\mathrm{a}}=2 \omega_{1}-\omega_{2}$. A 2 -mm-thick lithium triborate (LBO) crystal was used to convert the OPA output into second harmonic radiation. A tunable chirp was imposed on the laser pulses with an adjustable pulse stretcher, consisting of a pair of 600 -groove/mm diffraction gratings. Parameters of chirped pulses were retrieved from cross-correlation frequency-resolved optical gating measurements. The spectral measurements were performed with the use of fiber-optic spectrometers. A tunable delay time between the pulses was introduced by using a corner reflector mounted on a step-motor-driven stage. The laser pulses were brought into a spatial coincidence using a dichroic mirror and focused on a Raman sample with a microobjective with a numerical aperture $\mathrm{NA}=0.1$ and a magnification of 8 . The CARS signal was collected in the forward direction with an NA $=0.1, \times 4$ microobjective. This signal was separated from laser radiation by two short-pass and one band-pass filters and detected with an H9307-02 Hamamatsu photomultiplier tube. The electronic signal from PMT was amplified by an SR830 Stanford Research Systems lock-in amplifier with an integration time of $100 \mathrm{~ms}$.

1. Bloembergen, N. Nonlinear Optics (Benjamin, New York, 1965).

2. Shen, Y. R. The Principles of Nonlinear Optics (John Wiley \& Sons, New York, 1984).

3. Eesley, G. L. Coherent Raman Spectroscopy (Pergamon, Oxford, 1981).

4. Zheltikov, A. M. \& Koroteev, N. I. Coherent four-wave mixing in excited and ionized gas media. Phys. Uspekhi 42, 321-351 (1999).

5. Dudovich, N., Oron, D. \& Silberberg, Y. Single-pulse coherently controlled nonlinear Raman spectroscopy and microscopy. Nature 418, 512-514 (2002).

6. Baker, S., Walmsley, I. A., Tisch, J. W. G. \& Marangos, J. P. Femtosecond to attosecond light pulses from a molecular modulator. Nature Photonics 5, 664-671 (2011).

7. Pezacki, J. P. et al. Chemical contrast for imaging living systems: molecular vibrations drive CARS microscopy. Nature Chem. Biol. 7, 137-145 (2011).

8. Zumbusch, A., Holtom, G. R. \& Xie, X. S. Three-dimensional vibrational imaging by coherent anti-Stokes Raman scattering. Phys. Rev. Lett. 82, 4142-4145 (1999).

9. Freudiger, C. W. et al. Label-Free Biomedical Imaging with High Sensitivity by Stimulated Raman Scattering Microscopy. Science 322, 1857-1861 (2008).

10. Pestov, D. et al. Optimizing the laser-pulse configuration for coherent Raman spectroscopy. Science 316, 265-268 (2007).

11. Malevich, P. N. et al. Ultrafast-laser-induced backward stimulated Raman scattering for tracing atmospheric gases. Opt. Express 20, 18784-18794 (2012).

12. Zheltikov, A. M., Voronin, A. A., Kitzler, M., Baltuška, A. \& Ivanov, M. Optical detection of interfering pathways in subfemtosecond multielectron dynamics. Phys. Rev. Lett. 103, 033901 (2009).

13. Raman, C. V. \& Krishnan, K. S. A new type of secondary radiation. Nature 121, 501-502 (1928).

14. Zheltikov, A. M. Coherent anti-Stokes Raman scattering: from proof-of-theprinciple experiments to femtosecond CARS and higher order wave-mixing generalization. J. Raman Spectrosc. 31, 653-667 (2000).

15. Warren, W. S., Rabitz, H. \& Dahleh, M. Coherent Control of Quantum Dynamics: The Dream is Alive. Science 259, 1581-1589 (1993).

16. Zheltikov, A. M., Voronin, A. A., Kienberger, R., Krausz, F. \& Korn, G. Frequencytunable multigigawatt sub-half-cycle light pulses from coupled-state dynamics of optical solitons and impulsively driven molecular vibrations. Phys. Rev. Lett. 105, 103901 (2010).

17. Zheltikov, A. M. \& Naumov, A. N. High-resolution four-photon spectroscopy with chirped pulses. Quantum Electron. 30, 606-610 (2000).

18. Hellerer, T., Enejder, A. M. \& Zumbusch, A. Spectral focusing: High spectral resolution spectroscopy with broad-bandwidth laser pulses. Appl. Phys. Lett. 85, 25-27 (2004)

19. Rocha-Mendoza, I., Langbein, W. \& Borri, P. Coherent anti-Stokes Raman microspectroscopy using spectral focusing with glass dispersion. Appl. Phys. Lett. 93, 201103 (2008). 
20. von Vacano, B. \& Motzkus, M. Time-resolving molecular vibration for microanalytics: Single laser beam nonlinear Raman spectroscopy in simulation and experiment. Phys. Chem. Chem. Phys. 10, 681-691 (2008).

21. Lim, S.-H., Caster, A. G. \& Leone, S. R. Single-pulse phase-control interferometric coherent anti-Stokes Raman scattering spectroscopy. Phys. Rev. A 72, 041803 (2005)

22. Koroteev, N. I., Endemann, M. \& Byer, R. L. Resolved structure within the broadband vibrational line of liquid $\mathrm{H} 2 \mathrm{O}$ from polarization coherent anti-Stokes Raman spectroscopy. Phys. Rev. Lett. 43, 398-401 (1979).

23. van Rhijn, A. C. W., Jurna, M., Jafarpour, A., Herek, J. L. \& Offerhaus, H. L. Phaseshaping strategies for coherent anti-Stokes Raman scattering. J. Raman Spectrosc. 42, 1859-1863 (2011).

24. Jurna, M., Korterik, J. P., Otto, C., Herek, J. L. \& Offerhaus, H. L. Vibrational phase contrast microscopy by use of coherent anti-Stokes Raman scattering. Phys. Rev. Lett. 103, 043905 (2009).

25. Pegoraro, A. F. et al. Optimally chirped multimodal CARS microscopy based on a single Ti: sapphire oscillator. Opt. Express 17, 2984-2996 (2009).

26. Konorov, S. O. et al. Cross-correlation FROG CARS with frequency-converting PCFs. Phys. Rev. E 70, 057601 (2004).

27. Ivanov, A. A. et al. Coherent Raman spectroscopy with frequency-shifted and shaped pulses from a photonic-crystal fiber. Chem. Phys. Lett. 418, 19-23 (2006).

28. Lang, T. \& Motzkus, M. Single-shot femtosecond coherent anti-Stokes Ramanscattering thermometry. J. Opt. Soc. Am. B 19, 340-344 (2002).

29. Handbook of Raman Spectroscopy: From the Research Laboratory to the Process Line, ed. by Lewis, I. R. \& Edwards, H. G. M. (CRC Press, 2001).

30. Druet, S. A. J. \& Taran, J.-P. CARS spectroscopy. Progress Quantum Electron. 7, 1-72 (1981).
31. Jurna, M. et al. Visualizing resonances in the complex plane with vibrational phase contrast coherent anti-Stokes Raman scattering. Anal. Chem. 82, 7656-7659 (2010).

\section{Acknowledgments}

This work was supported in part by the Welch Foundation (grant no. A-1801), the Russian Foundation for Basic Research, and Skolkovo Foundation (grant no. 78).

\section{Author contributions}

A.A.L. designed and performed the experiments, analyzed the data, and prepared the graphic material. I.V.F. performed the experiments. A.B.F. and D.A.S.-B. participated in project planning and discussions of the results. A.M.Z. designed the experiments, analyzed the data, and wrote the paper.

\section{Additional information}

Competing financial interests: The authors declare no competing financial interests.

License: This work is licensed under a Creative Commons

Attribution-NonCommercial-NoDerivs 3.0 Unported License. To view a copy of this license, visit http://creativecommons.org/licenses/by-nc-nd/3.0/

How to cite this article: Lanin, A.A., Fedotov, I.V., Fedotov, A.B., Sidorov-Biryukov, D.A. \& Zheltikov, A.M. The phase-controlled Raman effect. Sci. Rep. 3, 1842; DOI:10.1038/ srep01842 (2013). 